\title{
Atherosclerosis of the right posterior hepatic artery in a patient with hilar cholangiocarcinoma undergoing left trisectionectomy: a case report of a therapeutic pitfall
}

Yuichi Goto* (D), Satoki Kojima, Yoriko Nomura, Daisuke Muroya, Syoichiro Arai, Hisamune Sakai, Ryuichi Kawahara, Toru Hisaka, Yoshito Akagi, Hiroyuki Tanaka and Koji Okuda

\begin{abstract}
Background: We experienced a rare case of benign arterial stricture of the right posterior hepatic artery (RPHA) caused by atherosclerosis in a patient with hilar cholangiocarcinoma.

Case presentation: A 75-year-old man was referred to our hospital for the detailed investigation of serum hepatobiliary enzyme elevation. The patient had a history of hypertension, type 2 diabetes mellitus, and an operative history of coronary artery bypass grafting 10 years before. Endoscopic retrograde cholangiography found strictures of the right and left hepatic ducts with involvement of right anterior and posterior bile ducts. Adenocarcinoma was evident by brush cytology. We diagnosed these findings as hilar cholangiocarcinoma and planned left trisectionectomy including bile duct reconstruction. Although the tumor and RPHA were not adjacent, preoperative multidetector computed tomography revealed a stricture of the RPHA that was $5.6 \mathrm{~mm}$ in length. We suspected that atherosclerosis caused the stricture, and we performed digital subtraction angiography and intravascular ultrasonography that showed stricture of the RPHA accompanied by thick plaques in the arterial wall. We placed a bare-metal stent in the RPHA and then performed left trisectionectomy. Since this patient developed bile leakage postoperatively, percutaneous drainage was performed. The bile leakage was successfully controlled, and the patient was discharged 3 months after surgery. Unfortunately, 4 months after hepatectomy, he was re-hospitalized with multiple pyogenic liver abscesses. We performed intensive multimodal treatment for the liver abscesses and stabilized the disease; however, we eventually lost this patient due to liver failure 14 months after surgery.

Conclusion: To the best of our knowledge, there is no previous literature on atherosclerosis of the RPHA, which was evident preoperatively in our case. Because arterial complications may lead to critical biliary complications in patients who undergo left trisectionectomy, we first performed prophylactic arterial stent placement. We speculate that existing chronic microscopic injury of the peribiliary plexus might have caused the liver abscesses. We successfully diagnosed atherosclerosis of the RPHA preoperatively. However, further investigation of patients is warranted to determine if left trisectionectomy is contraindicated in these patients.
\end{abstract}

Keywords: Hepatic artery, Stenosis, Atherosclerosis, Hilar cholangiocarcinoma, Pyogenic liver abscess

\footnotetext{
* Correspondence: gotou_yuuichi@med.kurume-u.ac.jp

Division of Hepatobiliary and Pancreatic Surgery, Department of Surgery,

Kurume University School of Medicine, 67 Asahi-machi, Kurume 8300011,

Japan
}

(c) The Author(s). 2018 Open Access This article is distributed under the terms of the Creative Commons Attribution 4.0 International License (http://creativecommons.org/licenses/by/4.0/), which permits unrestricted use, distribution, and reproduction in any medium, provided you give appropriate credit to the original author(s) and the source, provide a link to the Creative Commons license, and indicate if changes were made. The Creative Commons Public Domain Dedication waiver (http://creativecommons.org/publicdomain/zero/1.0/) applies to the data made available in this article, unless otherwise stated. 


\section{Background}

With the increasing number of elderly surgical candidates, underlying cardiovascular complications are an important factor for assessing the risk of surgery [1]. There is no previous literature on benign stricture of the right posterior hepatic artery (RPHA) caused by atherosclerosis. We report an extremely rare case of hilar cholangiocarcinoma, in which there was atherosclerosis of the RPHA. This patient was managed preoperatively by bare-metal stent placement followed by left trisectionectomy including Roux-en-Y choledochojejunostomy.

\section{Case presentation}

A 75-year-old male patient was referred to our hospital for the detailed investigation of elevated serum hepatobiliary enzymes. The patient had a history of hypertension, type 2 diabetes mellitus (DM) with diabetic nephropathy, malignant otitis externa, and an operative history of emergent coronary artery bypass grafting (CABG) 10 years before for myocardial infarction. During cardiac surgery, the right gastric artery, which was bifurcated from the common hepatic artery, was anastomosed to the posterior descending artery by the ante-gastric route. The height and weight of the patient was $1.62 \mathrm{~m}$ and $65 \mathrm{~kg}$, respectively. The performance status of the patient assessed by the Eastern Cooperative Oncology Group was Grade 1. The results of preoperative laboratory testing were as follows: white blood cell count, 5,900 cells $/ \mu \mathrm{L}$; red blood cell count, $478 \times 10^{4}$ cells $/ \mu \mathrm{L}$; serum hemoglobin concentration, $10.3 \mathrm{~g} / \mathrm{dL}$; serum platelet count, $16.6 \times 10^{4}$ platelets/ $\mu \mathrm{L}$; serum aspartate aminotransferase, $40 \mathrm{IU} / \mathrm{L}$; serum alanine aminotransferase, $32 \mathrm{IU} / \mathrm{L}$; serum alkaline phosphatase, $639 \mathrm{IU} / \mathrm{L}$; serum gamma glutamic transpeptidase, $297 \mathrm{IU} / \mathrm{L}$; total serum bilirubin, $0.66 \mathrm{mg} / \mathrm{dL}$; serum albumin, $3.91 \mathrm{~g} / \mathrm{dL}$; serum C-reactive protein, $0.05 \mathrm{mg} / \mathrm{dL}$; prothrombin time (\%), 114\%; hemoglobin A1c, 7.6\%; indocyanin green retention rate after $15 \mathrm{~min}, 4.0 \%$. The levels of serum carcinoembrionic antigen and carbohydrate antigen 19-9 were elevated at $2.5 \mathrm{ng} / \mathrm{mL}$ and $35.8 \mathrm{U} / \mathrm{mL}$, respectively.

Endoscopic retrograde cholangiography and 3-dimensional computed tomography cholangiography found abrupt narrowing of the common hepatic duct that was $20 \mathrm{~mm}$ in length including the right and left hepatic ducts, with involvement of the right anterior and posterior bile duct bifurcations (Fig. 1a-d). Adenocarcinoma was evident by the cytological findings, and this was confirmed by brush cytology. Contrast enhanced computed tomography (CECT) showed no distant metastases or lymph node metastases. We diagnosed these findings as Bismuth type 4, T2N0M0 Stage II hilar cholangiocarcinoma. According to the preoperative imaging findings, the left bile duct was completely occluded by cancer, there was narrowing of right hepatic duct and anterior sector bile duct, and only the right

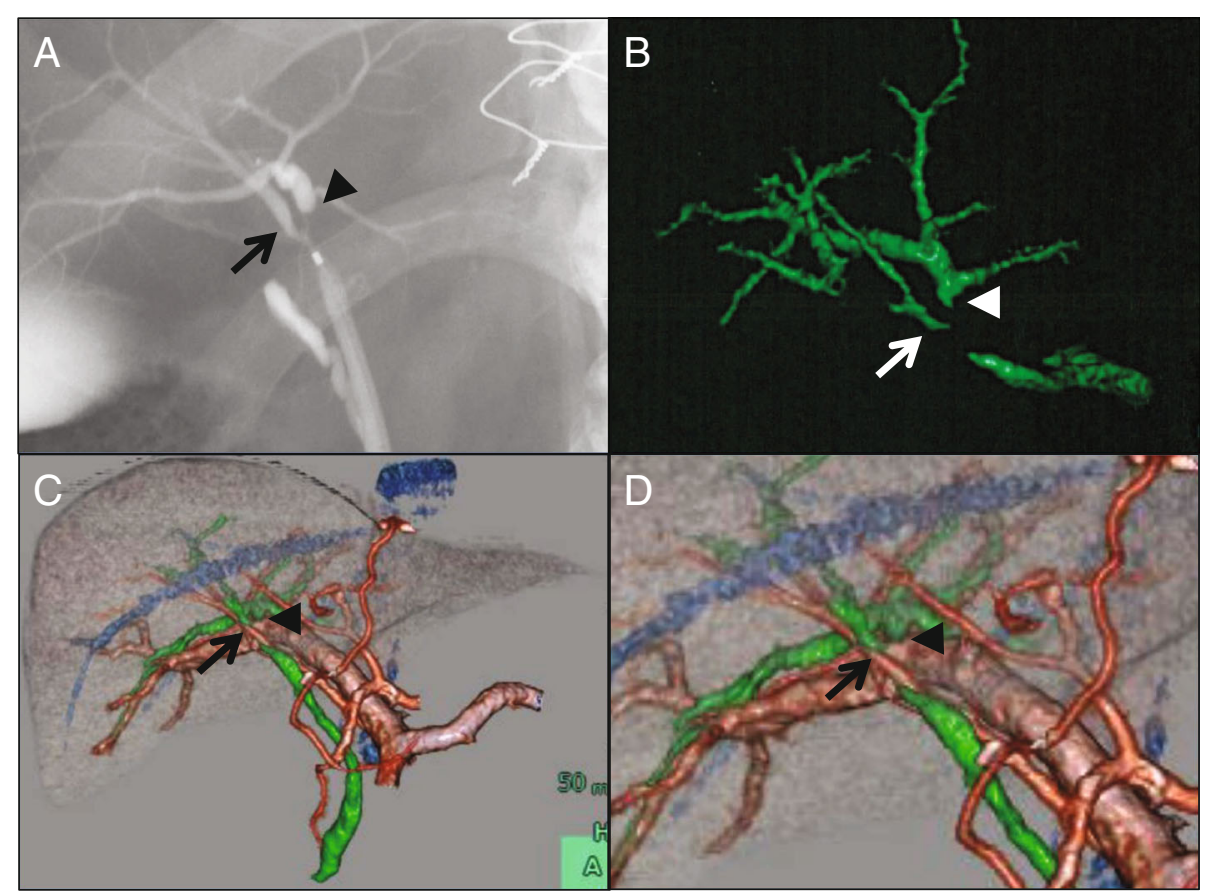

Fig. 1 Endoscopic retrograde cholangiography (a) and 3-dimensional CT cholangiography (b, c, d) reveals stenosis of the common hepatic duct that was $20 \mathrm{~mm}$ in length including the right and left hepatic ducts, with involvement of the right anterior (arrow) and posterior (arrow head) bile duct bifurcation. The pattern of confluence of the right posterior hepatic artery is of the supra-portal type. The left hepatic duct is completely occluded by cancer and could not be identified 
posterior bile duct was free from the margin. Therefore, we planned left trisectionectomy, extrahepatic bile duct resection, and Roux-en-Y choledochojejunostomy.

CECT also showed that the right hepatic artery was bifurcated from the supra mesenteric artery, and the left hepatic artery was dominant from the celiac artery. The bile duct, which was involved in the cholangiocarcinoma, and the RPHA were not adjacent (the distance between them was $8 \mathrm{~mm}$ ) because the pattern of confluence of the right posterior hepatic duct was of the supra-portal type, although the narrowing of the RPHA was $5.6 \mathrm{~mm}$ in length. Because this patient had multiple cardiovascular risk factors including a history of CABG, we suspected that the narrowing of the RPHA was a benign stricture caused by atherosclerosis. Arterial imaging with 3-dimensional multidetector computed tomography (MDCT) (Fig. 2a) and curved planner reconstruction (CPR) of MDCT also showed stricture of the RPHA (Fig. 2b). We performed digital subtraction angiography (DSA) and intravascular ultrasonography (IVUS). DSA showed stricture of the RPHA (Fig. 3a), and thick plaques in the arterial intima were evident on IVUS (Fig. 3b). According to these findings, we diagnosed stricture of the RPHA due to atherosclerosis, and we performed percutaneous balloon arterioplasty followed by bare-metal stent placement to prevent postoperative biliary complications (Fig. 4a-d). His clinical course after stent placement was uneventful, and we performed left trisectionectomy, extrahepatic bile duct resection, and Roux-en-Y choledochojejunostomy 51 days after stent placement.
Since this patient developed bile leakage postoperatively, percutaneous drainage was performed. The bile leakage was successfully controlled without stenosis of the choledochojejunostomy (Fig. 5a, b), and the patient was discharged 3 months after surgery. Unfortunately, 4 months after hepatectomy, he was re-hospitalized with multiple pyogenic liver abscesses (Fig. 6a). Klebsiella pneumoniae was identified from the liver abscess, and we performed intensive multimodal treatment for the pyogenic liver abscesses. Although we stabilized the disease (Fig. 6b), we eventually lost this patient due to liver failure 14 months after surgery.

\section{Discussion and conclusion}

Left trisectionectomy is the treatment of choice for patients with Bismuth type 4 hilar cholangiocarcinoma. Although the safety of this procedure has been increasing, the reported incidence of postoperative complications is still high [2-4]. Because the RPHA is the only remaining artery that supplies blood flow to the intrahepatic bile duct after left trisectionectomy, preoperative assessment of RPHA is important. To the best of our knowledge, there is no previous literature on atherosclerosis of the RPHA, and there is no consensus about how it should be managed.

Since our patient had hilar cholangiocarcinoma that was accompanied by benign stricture of the RPHA due to atherosclerosis, we performed stent placement before left trisectionectomy. To assess vascular anatomy and liver volume, we performed 128-slice MDCT. MDCT in

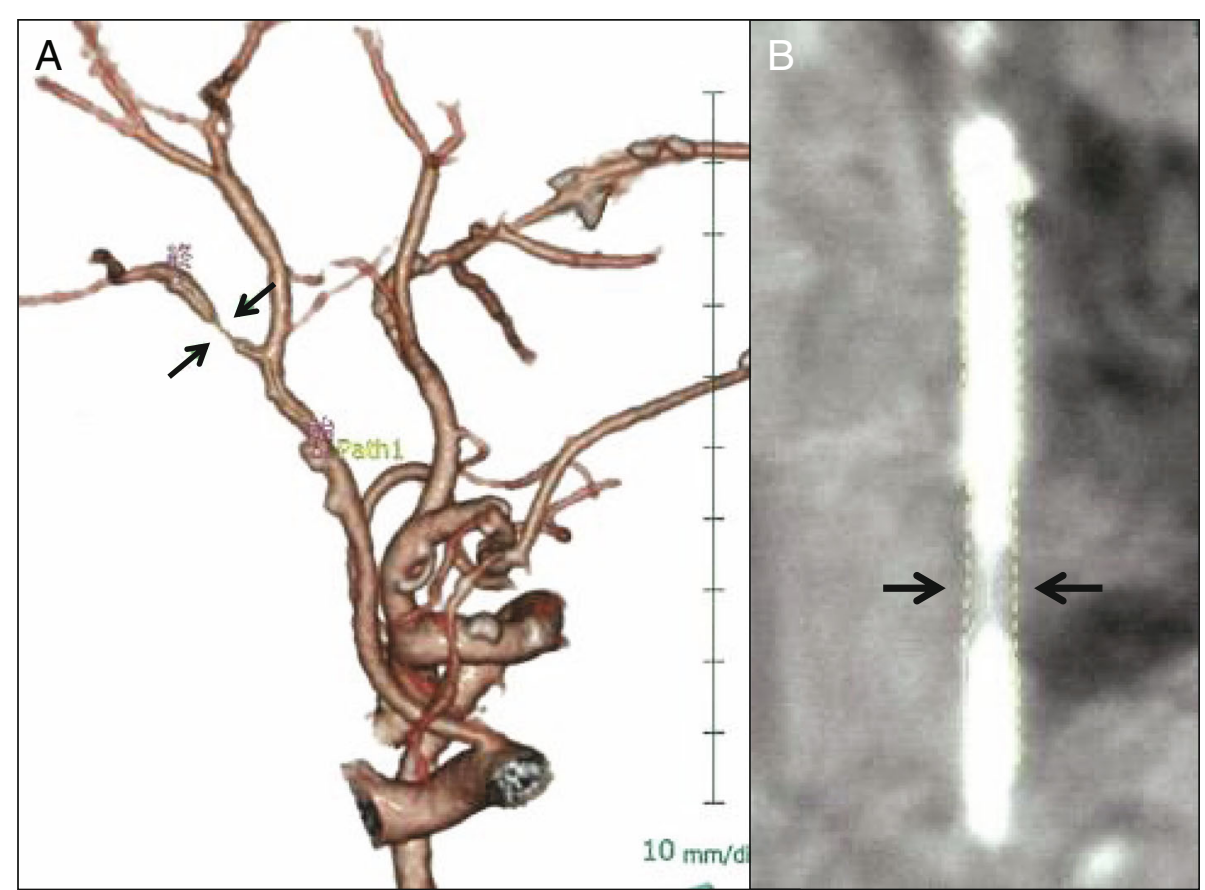

Fig. 2 Three-dimensional multidetector computed tomography (MDCT) (a) and curved planner reconstruction of MDCT (b) showed stricture of the right posterior hepatic artery that was $5.6 \mathrm{~mm}$ in length (arrows) 


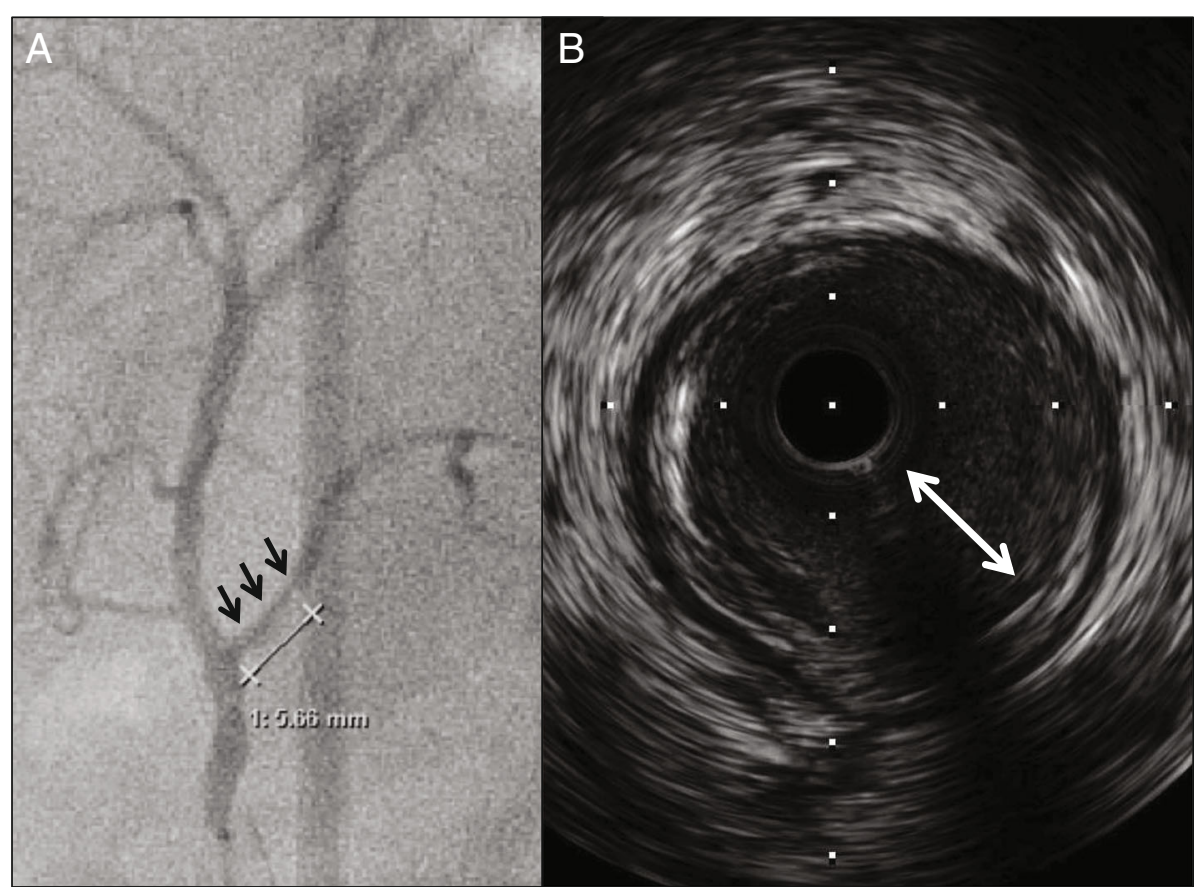

Fig. 3 Digital subtraction angiography (a) shows stricture of the right posterior hepatic artery (arrows), and thick plaques (double-head arrow) in the arterial intima were observed by intravascular ultrasonography (b)

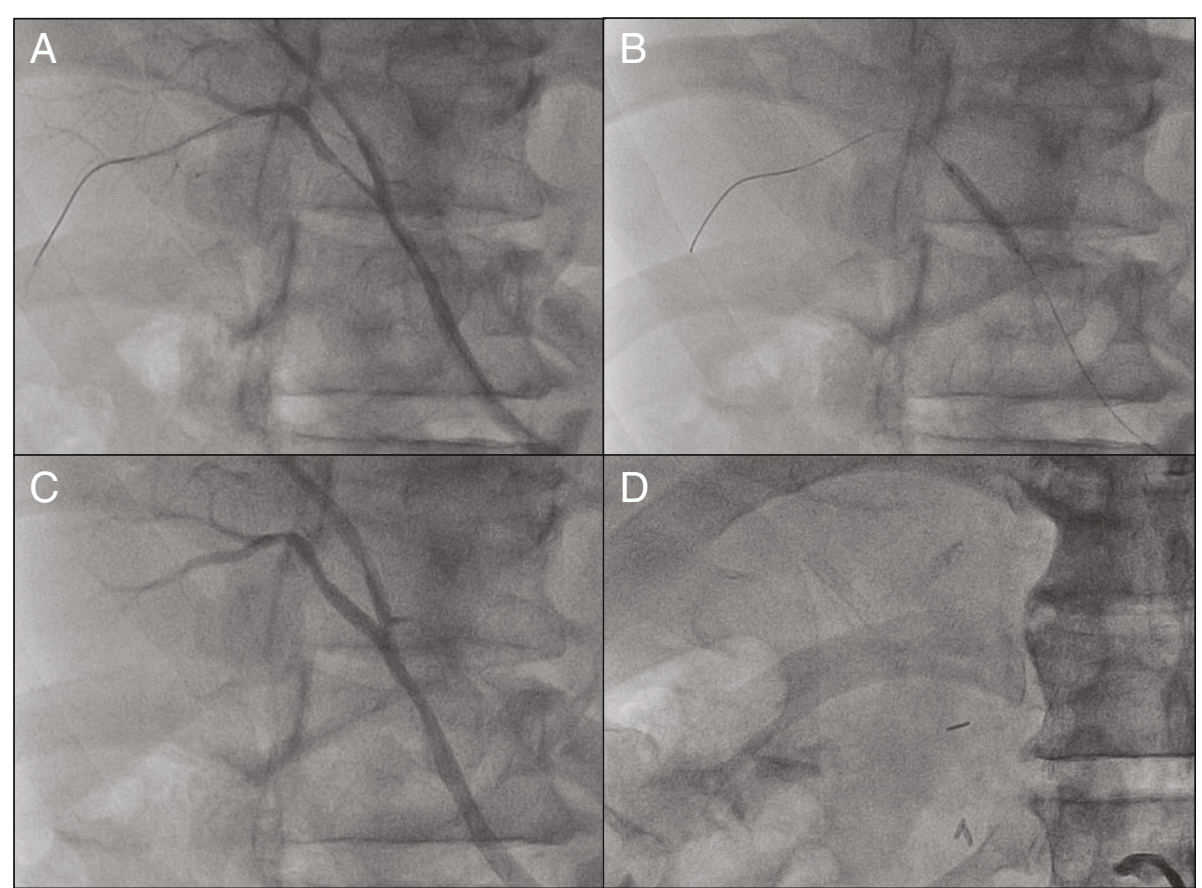

Fig. 4 Digital subtraction angiography before (a) and after the percutaneous balloon arterioplasty (b). The stricture of the right posterior hepatic artery was improved after placement of the bare-metal stent $(\mathbf{c}, \mathbf{d})$ 


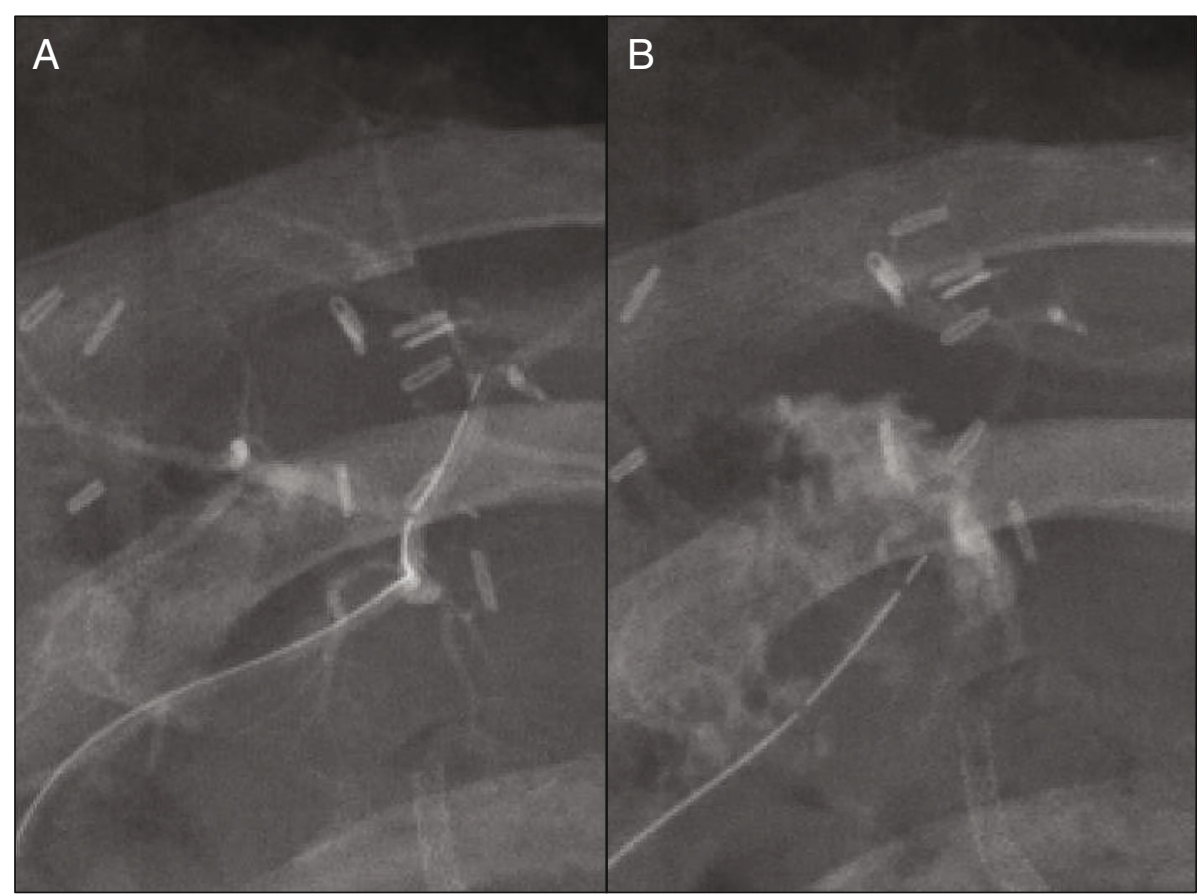

Fig. 5 Cholangiography of the bile duct drainage tube revealed no stenosis of the choledochojejunostomy. There was no dilatation of the posterior bile duct (a), and the passage of contrast media was smooth without retention after removal of the drainage tube (b)
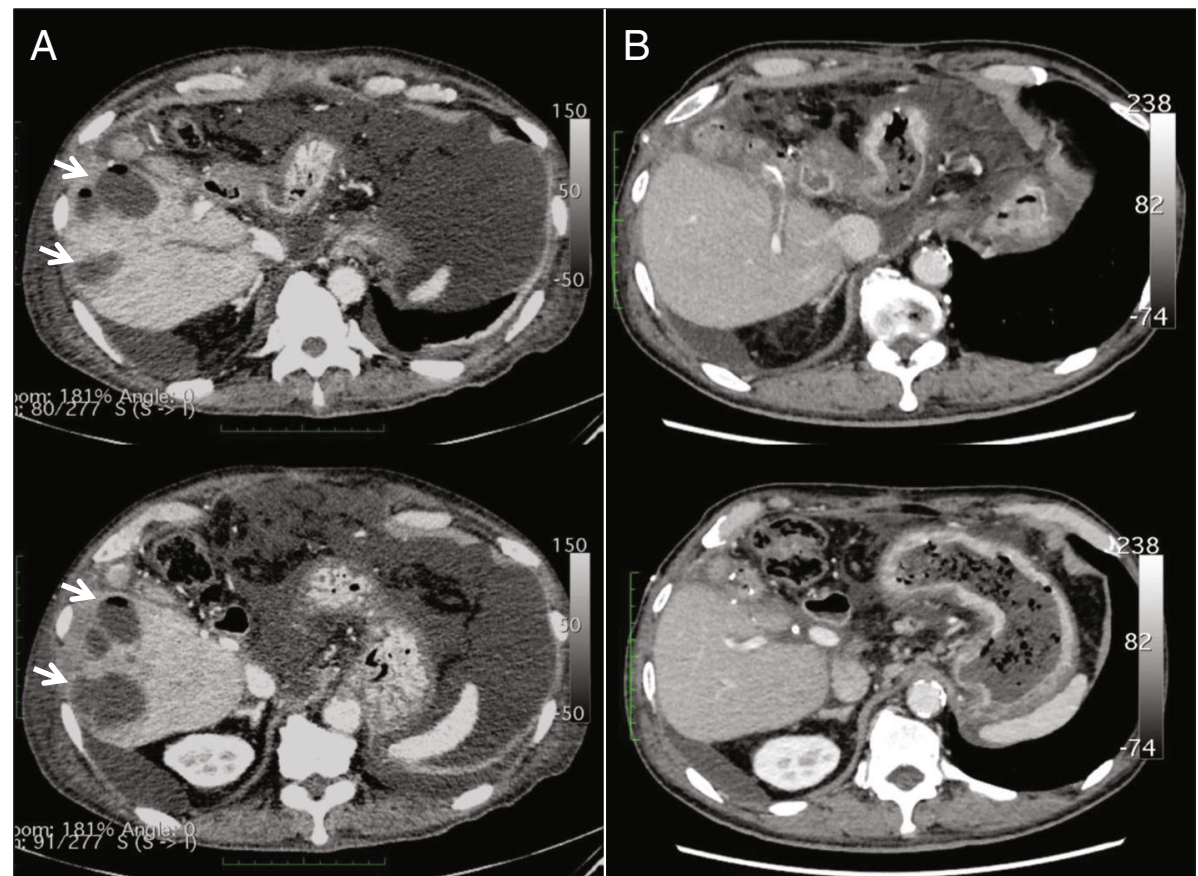

Fig. 6 Multiple pyrogenic liver abscesses (arrows) in the remnant liver (a). Liver abscesses disappeared after intensive treatment (b) 
combination with CPR analysis was quite sensitive to identify stenosis of the RPHA, and atherosclerosis was confirmed by IVUS. Because it has been reported that atherosclerosis of the hepatic artery is related to arterial dissection, aneurysm, and thrombosis [5], we performed prophylactic stent placement to prevent postoperative arterial complications. Although the diagnostic and therapeutic procedures were successful, the patient suffered from multiple pyogenic liver abscesses 4 months after liver surgery. The incidence of postoperative pyogenic liver abscess in patients with hilar cholangiocarcinoma undergoing major hepatectomy is reported to range from 20 to $37.5 \%[4,6]$. Concomitant resection of the hepatic artery and/or portal vein accompanied by vascular reconstruction is associated with a high risk of liver abscess after surgery [6]. Biliary disorders including cholecystolithiasis, choledocholothiasis, and hepatolithiasis are also related to liver abscess [6], although they were not evident in our case.

From an anatomical perspective, the intrahepatic biliary ducts are surrounded by a rich microvascular network, which is called the peribiliary plexus [7]. Some experimental studies suggested that there is an arterioportal communication through the peribiliary plexus [8]. The peribiliary plexus represents a collateral source of arterial blood to the liver when the hepatic artery is occluded [9]. We also performed Doppler ultrasonography to measure the resistive index of the RPHA and confirmed favorable arterial blood flow before and after stent placement including liver surgery. When the patient was re-hospitalized for pyogenic liver abscesses, there was no sign of arterial complications including stent occlusion. Since arterial flow through the RPHA was preserved, we speculate that microscopic injury of the peribiliary plexus including ischemic changes possibly due to atherosclerosis might have caused his pyrogenic liver abscesses. Since DM has also been reported to be associated with liver abscess in patients with hilar cholangiocarcinoma who undergo surgery, it possible that DM could have influenced the outcome of our patient, even though his DM was controlled by insulin therapy $[10,11]$. In addition, our patient also suffered from refractory peptic ulcer and was treated with a proton-pump inhibitor (PPI). Prolonged use of a PPI has been reported to increase the risk and mortality of pyogenic liver abscess [12]. Since our patient had vascular complications as well as other comorbidities that are associated with liver abscess, it possible that all of these factors could have affected the outcome.

As for the stent placement, a drug-eluting stent might be better than a bare-metal stent for reducing the risk of stent thrombosis [13]. One reason that we selected a bare-metal stent was that we had to cover the artery from the right hepatic artery to the RPHA without disturbing arterial flow of the right anterior hepatic artery, since the stenosis of the RPHA extended to the bifurcation of the anterior and posterior hepatic arteries. Another reason was the timing of liver surgery. After stent placement, double antiplatelet therapy is routinely administered, and the American College of Cardiology and American Heart Association guidelines recommended postponing elective non-cardiac surgery until 30 days after bare-metal stent placement and 6 months after drug-eluting stent placement [14]. We finally performed left trisectionectomy 51 days after placement of the bare-metal stent, and we do not think it would have been possible to wait for 6 months, since delay of surgery in patients with hilar cholangiocarcinoma will reduce the cure rate due to disease progression.

There is a limitation of our report. Since there was no autopsy in this patient after his death, histopathological assessment of the remnant liver was not performed to determine if there was microvascular injury of the peribiliary plexus due to atherosclerosis.

We successfully diagnosed atherosclerosis of the RPHA preoperatively by multiple imaging modalities and performed stent placement to prevent postoperative complications; however, we lost the patient due to the pyogenic liver abscesses. Further investigations are warranted to confirm if atherosclerosis of the RPHA is a potential risk factor for liver abscess and to determine if left trisectionectomy for hilar cholangiocarcinoma is contraindicated in these patients.

\section{Abbreviations \\ CABG: Coronary artery bypass graft; CECT: Contrast enhanced computed tomography; DM: Diabetes mellitus; DSA: Digital subtraction angiography; ERCP: Endoscopic retrograde cholangiography; IVUS: Intravascular \\ ultrasonography; MDCT: Multidetector computed tomography; RPHA: Right posterior hepatic artery}

\section{Ethical approval and consent to participate}

Written informed consent was obtained from the widow of the patient for participate of this Case report and any accompanying images. A copy of the written consent is available for review by the Editor of this journal. This is retrospective case report. For this type of study, ethics approval was waived by Ethics Committee of Kurume University Hospital.

Availability of data and materials

All the data supporting our findings is contained within the manuscript.

\section{Authors' contributions}

YG and SK collected the data and wrote this paper. YN, DM, SA, HS, RK, TH, $Y A, H T$, and $K O$ contributed to the study concept and review of the final manuscript. All authors read and approved the final manuscript.

\section{Consent for publication}

Written informed consent was obtained from the widow of the patient because this patient died of liver failure before we started writing this paper. A copy of the written consent is available for review by the Editor of this journal.

Competing interests

The authors declare that they have no competing interests. 


\section{Publisher's Note}

Springer Nature remains neutral with regard to jurisdictional claims in published maps and institutional affiliations.

Received: 8 March 2018 Accepted: 18 September 2018

Published online: 24 September 2018

\section{References}

1. Russell MC. Complications following hepatectomy. Surg Oncol Clin N Am. 2015;24:73-96.

2. Govil S, Bharatan A, Rammohan A, Kanagavelu R, Kaliamoorthy Reddy MS, et al. Liver resection for perihilar cholangiocarcinoma why left is sometimes right. Hpb [Internet]. International HepatoPancreato-Biliary Association Inc.; 2016;18:575-9. Available from: https://doi.org/10.1016/j.hpb.2016.05.002

3. Igami T, Nishio H, Ebata T, Yokoyama Y, Sugawara G, Nimura Y, et al. Surgical treatment of hilar cholangiocarcinoma in the "new era": the Nagoya University experience. J Hepatobiliary Pancreat Sci. 2010;17:449-54.

4. Hirano S, Kondo S, Tanaka E, Shichinohe T, Tsuchikawa T, Kato K, et al. Outcome of surgical treatment of hilar cholangiocarcinoma: a special reference to postoperative morbidity and mortality. J Hepatobiliary Pancreat Sci. 2010;17:455-62.

5. Karaosmanoglu D, Erol B, Karcaaltincaba M. Pictorial essay multidetector ct of hepatic artery pathologies. Jbr-Btr. 2012;95:345-9.

6. Zhang C, Li T, Chen Z, Chen Q, Zhi X. ljcem0013769Pdf. 2015;8:21279-86.

7. Terada T, Ishida F, Nakanuma Y. Vascular plexus around intrahepatic bile ducts in normal livers and portal hypertension. J Hepatol. 1989;8:139-49.

8. Cho KJ, Lunderquist A. The peribiliary vascular plexus: the microvascular architecture of the bile duct in the rabbit and in clinical cases. Radiology [Internet]. 1983;147:357-64. Available from: http://www.ncbi.nlm.nih.gov/pubmed/6836115

9. Stapleton GN, Hickman R, Terblanche J. Blood supply of the right and left hepatic ducts. Br J Surg. 1998;85:202-7.

10. Lai KC, Cheng KS, Bin JL, Huang CC, Lee YT, Chang HR, et al. Factors associated with treatment failure of percutaneous catheter drainage for pyogenic liver abscess in patients with hepatobiliary-pancreatic cancer. Am J Surg. 2013;205:52-7.

11. Law ST, Li KK. Is pyogenic liver abscess associated with recurrent pyogenic cholangitis a distinct clinical entity? A retrospective analysis over a 10-year period in a regional hospital. Eur J Gastroenterol Hepatol. 2011;23:770-7.

12. Bettinger D, Martin D, Rieg S, Schultheiss M, Buettner N, Thimme R, et al. Treatment with proton pump inhibitors is associated with increased mortality in patients with pyogenic liver abscess. Aliment. Pharmacol. Ther [Internet]. 2018;56. Available from: http://doi.wiley.com/10.1111/apt.14512

13. Palmerini T, Biondi-Zoccai G, Della RD, Stettler C, Sangiorgi D, D'Ascenzo F, et al. Stent thrombosis with drug-eluting and bare-metal stents: evidence from a comprehensive network meta-analysis. Lancet. 2012;379:1393-402.

14. Childers CP, Maggard-Gibbons M, Ulloa JG, MacQueen IT, Miake-Lye IM, Shanman $\mathrm{R}$, et al. Perioperative management of antiplatelet therapy in patients undergoing non-cardiac surgery following coronary stent placement: a systematic review. Syst. Rev. [Internet]. Systematic Reviews; 2018;7:4. Available from: https://systematicreviewsjournal.biomedcentral. com/articles/10.1186/s13643-017-0635-z

Ready to submit your research? Choose BMC and benefit from:

- fast, convenient online submission

- thorough peer review by experienced researchers in your field

- rapid publication on acceptance

- support for research data, including large and complex data types

- gold Open Access which fosters wider collaboration and increased citations

- maximum visibility for your research: over $100 \mathrm{M}$ website views per year

At $\mathrm{BMC}$, research is always in progress.

Learn more biomedcentral.com/submissions 